Acta Mechanica 13, 31-53 (1972)

(c) by Springer-Verlag 1972

\title{
A Constitutive Representation for Linear Aging, Environmental-Dependent Viscoelastic Materials
}

\author{
By \\ D. C. Stouffer, Cincinnati, Ohio, and A. S. Wineman, Ann Arbor, Michigan
}

With 2 Figures

(Received April 20, 1970)

\section{Summary - Zusammenfassung}

A Constitutive Representation for Linear Aging, Environmental-Dependent Viseoelastic Materials. This study is concerned with developing a constitutive law for aging and environmental-dependent materials. The development rests on the assumption that the mechanical properties of the aforementioned materials can be mathematically represented by a functional of the strain and environmental histories, and this functional depends on the present time and the time the material is created. This constitutive assumption leads to two equivalent integral forms of the constitutive law after asserting that the functional is linear in the strain history. The first form of the integral law contains a material response functional analogous to the creep and relaxation functions of classical linear viscoelasticity. The second integral law has the same basic mathematical form but the physical interpretation of the mechanical response functional is different. It is demonstrated that both forms of the aging law reduce to the same non-aging law which is the usual starting for an analysis of only environmentaldependent materials. In the Appendix an operator algebra is presented for convenience in manipulating the integral laws for aging materials.

Ein Werkstoffgesetz für lineares Altern und für umgebungsabhängige viskoelastische Stoffe. Diese Untersuchung beschäftigt sich mit der Darstellung eines Werkstoffgesetzes für Altern und für umgebungsabhängige Stoffe. Die Entwicklung beruht auf der Annahme, daß die mechanischen Eigenschaften der oben erwähnten Stoffe mathematisch durch ein Funktional der Verzerrungs- und der Umgebungsgeschichten dargestellt werden kann und $\mathrm{daB}$ dieses Funktional von der augenblicklichen Zeit und der Zeit der Erzeugung des Stoffes abhängt. Diese Werkstoffannahme führt, unter der Voraussetzung einer linearen Abhängigkeit des Funktionals von der Verzerrungsgeschichte, auf zwei äquivalente Integraldarstellungen des Werkstoffgesetzes. Die erste Darstellung des Integralgesetzes enthält ein MaterialantwortFunktional analog der Kriech- und Relaxationsfunktionen der klassischen linearen Viskoelastizität. Das zweite Gesetz hat die gleiche mathematische Gestalt, aber verschiedene physikalische Interpretation des mechanischen Antwort-Funktionals. Es wird gezeigt, daß für nicht-alternde Stoffe beide Darstellungen dasselbe Gesetz, das der übliche Ausgangspunkt einer Untersuchung umgebungsbeeinflußter Stoffe ist, ergeben. Im Anhang werden einige für das Rechnen mit den Integralgesetzen alternder Stoffe handliche Sätze der Operatorenrechnung angegeben.

\section{Introduction}

The integral constitutive equation for linear viscoelasticity can be obtained heuristically by standard arguments for constructing superposition integrals. The derivation of such laws for linear non-aging environmental independent response 
was put on a firm theoretical basis in 1962 by GURTIN and STERnBERG [1]. They made fundamental assumptions about the constitutive equation which enabled them to deduce its integral representation from appropriate theorems. In 1960, MoRLAND and LeE [2] developed a constitutive equation for linear non-aging response which can be influenced by variations in temperatures. After developing a representation for the relaxation function for a certain class of materials which includes the effect of temperature variation, they constructed the integral constitutive law by the usual superposition arguments. Recently, SToufFER and WINEMAN [3] considered the general theory of the constitutive equation for linear non-aging materials whose response is effected by variations in the environment. Basic assumptions were formulated from which the integral representation for the constitutive law was deduced. In this way, the existence of a environmental dependent relaxation function was shown. A possible form of this dependence was derived for a class of materials which includes that considered by MoRLAND and LeE.

The theory of the constitutive equation for linear aging materials appears to be less developed. The constitutive equations for aging materials presented in the book by ARUTYUNYAN [4] and the excellent review by SACKMAN [5] are derived using heuristic superposition integral arguments. SACKMAN briefly discussed the influence of environment but did not include its effect in the constitutive equation. The purpose here is to lay down basic assumptions for linear aging viscoelastic materials, allowing for environmental influence, and then deduce the constitutive equations.

The development rests on the constitutive assumption given in section 2 that the stress tensor is a functional of the strain and environment histories, and depends explicitly on the current time and the time the material is created. A trivial application of the time shift aspect of the Principle of Material Frame Indifference shows that there are two equivalent forms for the constitutive equation. In one case, the strain and environmental histories are referred to creation time and in the second they are referred to current time. In section 3, it is assumed that the response functionals are linear in the strain history. This property is first used to establish tensorial properties for the response functional and derive restrictions due to isotropy. It is then used to obtain two equivalent integral representations for the constitutive equation. The physical interpretations of the response functions introduced here are discussed in section 4 , along with relations between creep and relaxation properties. For the kind of aging materials for which this theory may be useful, i.e. curing concrete, the 'aging' constitutive equation should approach a 'non-aging' constitutive law for large times.

This is easily accounted for, in section 5, by introducing a time beyond which the material response is translation-invariant [1]. That is, after a certain time, if two strains (and environmental) histories differ only by a shift in time, then their corresponding stress histories differ only by that shift. This treats the concept of translation invariance as a property of a kind of material response rather than a version of the Principle of Material Frame Indifference. The landmark work of GURTIN and STERNBERG [1] introduced an operator algebra for the integral constitutive law which has greatly simplified the manipulations in problems in non-aging environmental independent theory. In the Appendix analogous operator 
algebras are presented for convenience in manipulating either form of integral law for aging materials.

One final comment should be made regarding this study. The constitutive assumption recognizes that the environmental phenomena may chemically react with the aging material to alter the mechanical properties. The results and diseussion may then be regarded as applying to a constitutive equation derived from a theory of chemically reacting mixtures. It is hoped however that such a complicated theory may not be necessary for some aging materials, such as perhaps euring concrete. Instead, relaxation or creep tests could be carried out in various steady environments. Then, just as for non-aging environmental dependent materials, the response during time varying environments can be inferred.

\section{The General Constitutive Relationship}

There are several time variables which can appear in any constitutive assumption. In this section the manner in which constitutive equation depends on these variables is investigated. To begin, denote a sequence of times which are measured relative to an arbitrary origin. Let

$t_{c}$ represent the creation time of the material,

$t$ represent the current time, and

$\tau$ represent the running time variable $\left(t_{c} \leq \tau \leq t\right)$.

Time $t_{c}$ also denotes an appropriate beginning of any aging phenomenon that may be relevant to the particular material and process under consideration. This may be the time when the material is made. On the other hand, for problems involving concrete or glue, it may be more suitable to consider $t_{c}$ as the time when the material is cast into some shape or spread in a glue line. In any case, $t_{c}$ is the first reference in time and no event prior to $t_{c}$ is considered relevant.

Let $\sigma_{i j}(\boldsymbol{x}, t)$ and $\varepsilon_{i j}(\boldsymbol{x}, t)$ be the components in the same Cartesian coordinate system of the stress and infinitesimal strain tensors of a particle occupying position $x$ at time $t$ in body $B$ and denote them by $\sigma$ and $\varepsilon$ respectively. Let the components of $\sigma$ and $\varepsilon$ be defined and continuous on $\left[t_{c}, \infty\right)$. The notation $[a, b]$ represents the closed interval with the endpoints $a$ and $b ;(a, b)$ is the open interval without the endpoints $a$ and $b ;[a, b)$ and $(a, b]$ denotes the mixed intervals open on the right and left ends respectively.

Next, consider the environmental parameters that can influence the mechanical response of a material; for example, temperature, humidity, concentration, radiation intensity, etc. Let $\varphi(\boldsymbol{x}, t)$ represent the set of all environmental properties of the particle at position $\boldsymbol{x}$ in body $B$. Assume $\varphi(x, t)$ is defined and continuous for all $t$ in the interval $\left[t_{c}, \infty\right)$. The functions $\sigma, \varepsilon$, and $\varphi$ will be called the stress, strain and environmental histories respectively.

Assumption 1. The stress at any given particle $\boldsymbol{x}$ in body $B$ at some time $t$ is completely determined by the mechanical strain and environmental histories at the particle on the interval $\left[t_{c}, t\right]$, and depends on the present time $t$ and the creation time $t_{c}$ of the material. Furthermore, if the mechanical strain $\varepsilon=0$ on the interval $\left[t_{c}, t\right]$, then $\sigma=\mathbf{0}$ on $\left[t_{c}, t\right]$. 
The dependence of $\sigma$ on the histories confined to the time interval $\left[t_{c}, t\right]$ incorporates the property of non-retroactivity which is part of the constitutive definition of GuRTIN and STERNBERG ([1], p. 303). Further it will be assumed that any strain history may be associated with any environmental history. Any coupling that does occur can be accounted for in the appropriate field equations. Assumption 1 can be characterized by the equation

$$
\sigma(t)=\mathscr{F}\left[\begin{array}{cc}
t & t \\
\varepsilon\left(\tau_{1}\right) ; \varphi\left(\tau_{2}\right) ; t, t_{c} \\
\tau_{\mathbf{1}}=t_{c} & \underset{\tau_{2}=t_{c}}{c}
\end{array}\right]
$$

where $\mathfrak{F}$ is a functional relating a stress history $\sigma(t)$ to each strain and environmental history $\varepsilon(t)$ and $\varphi(t)$ for every $t$ and $t_{c}$ in $(-\infty, \infty)$. The quantity $\varepsilon(t)$ represents the mechanical strain history.

The constitutive Equation (2.1) contains two time variables $t_{c}$ and $t$ to represent the aging phenomenon of the material. To determine how these variables are relevant to the model the Principle of Material Frame-Indifference ([6], p. 22) can be applied to (2.1). Since the current study is restricted to infinitesimal deformations and is concerned with primarily the temporal aspects of material response only the time-translation portion of the frame-transformation is considered here. This restriction embodies the principle that in a scale transformation of all events, time intervals and the sense of time are preserved.

In order to impose the restriction of Material Frame-Indifference, consider a second set of events occurring $a$ time units earlier than the first. The significant time variables are given by:

$$
\left(t^{\prime}=t-a\right), \quad\left(\tau_{n}{ }^{\prime}=\tau_{n}-a\right) \quad \text { and } \quad\left(t_{c}{ }^{\prime}=t_{c}-a\right) ; \quad(n=1,2),
$$

while the strain and environmental histories are defined by

and

$$
\bar{\varepsilon}\left(t^{\prime}\right)=\bar{\varepsilon}(t-a)=\varepsilon(t)
$$

$$
\bar{\varphi}\left(t^{\prime}\right)=\bar{\varphi}(t-a)=\varphi(t) .
$$

The stress at time $t^{\prime}$ can be determined from Equation (2.1) as

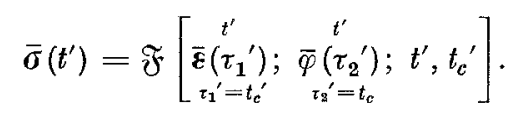

The Principle of Material Frame-Indifference requires

$$
\sigma(t)=\bar{\sigma}\left(t^{\prime}\right)
$$

Equation (2.5) together with (2.1) and (2.4) establishes a restriction on the function $\mathfrak{F}$

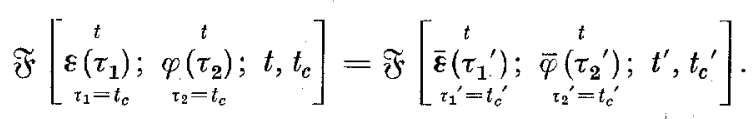

Equation (2.6) can give rise to two representations of the functional $\mathfrak{F}$. 

Then,

First let $a=t$ in Equation (2.2). This refers all events to the current time.

$$
t^{\prime}=0, \quad \tau_{n}{ }^{\prime}=\tau_{n}-t \quad \text { and } \quad t_{c}^{\prime}=t_{c}-t ; \quad(n=1,2) .
$$

Using (2.3), Equation (2.6) becomes

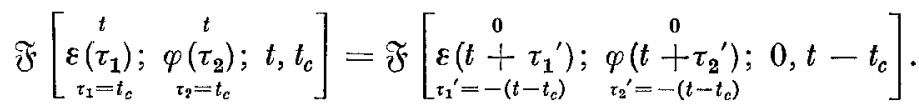

Defining $\tau_{n}{ }^{\prime}=-s_{n}(n=1,2)$ and $\mathfrak{F}$ to be a new functional, the constitutive equation becomes

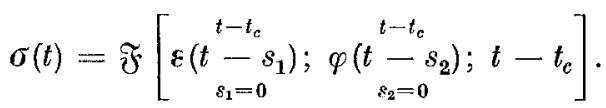

The second form can be derived by letting $a=t_{c}$, i.e., referring all events to the creation time. Equation (2.2) becomes

$$
t^{\prime}=t-t_{c}, \quad \tau_{n}{ }^{\prime}=\tau_{n}-t_{c}, \quad \text { and } \quad t_{c}{ }^{\prime}=0 ; \quad(n=1,2) .
$$

Using (2.3) Equation (2.6) can now be written as

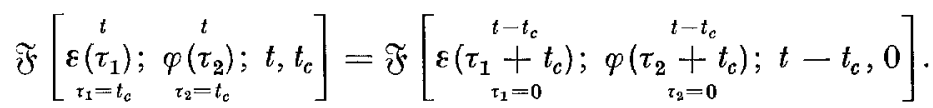

Again, defining $\mathfrak{F}$ to be a second new functional, then

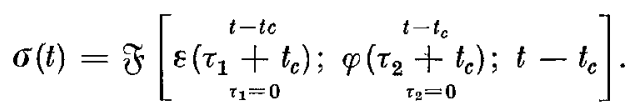

In Equations (2.8) and (2.10) the time transformation demonstrates the fact for measuring material response there is no specific time origin. Thus, the Principle of Material Frame-Indifference is satisfied if the resulting functionals depend on the time elapsed since creation $t-t_{c}$ (that is, the "age of material"), and the strain and environmental states occurring during this elapsed time. Since the functionals depend only on the quantities measured between $t_{c}$ and $t, t_{c}$ can be set equal to zero with no loss in generality. Therefore Equation (2.1) can now be written as

or

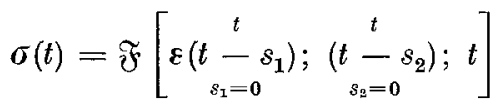

$$
\sigma(t)=\mathscr{F}\left[\begin{array}{cc}
t & t \\
\varepsilon\left(\tau_{1}\right) ; & \varphi\left(\tau_{2}\right) ; t \\
\tau_{1}=0 & \tau_{2}=0
\end{array}\right] \text {. }
$$

Equations (2.11) and (2.12) describe two equivalent forms of the stress-strain law. In Equation (2.11) the arguments of the strain and environmental histories are measured relative to the present time. In Equation (2.12) the arguments of $\varepsilon$ and $\varphi$ are measured relative to the creation time. For both equations $t$ denotes the age of the material, since $t_{c}$ was chosen as the origin of the time scale. Also note that the last part of assumption 1 now states that if $\varepsilon=0$ on $[0, t]$ then $\sigma=0$ on $[0, t]$. 


\section{Integral Representations for the Constitutive Equation}

If the response functional $\mathscr{F}$ of either $(2.11)$ or $(2.12)$ satisfies the following assumption of linearity in the strain history, then it can be shown that $\mathscr{F}$ is a fourth order tensor functional and has two integral representations.

Assumption 2. For the phenomena and materials to be considered, $\widetilde{F}$ is assumed to be linear in the strain history. That is, for every pair of real constants $\alpha_{1}$ and $a_{2}$ and each fixed environmental history $\varphi$,

$$
\mathfrak{F}\left[a_{1} \varepsilon_{1}+a_{2} \varepsilon_{2} ; \varphi, t\right]=a_{1} \mathfrak{F}\left[\varepsilon_{1} ; \varphi ; t\right]+a_{2} \mathfrak{F}\left[\varepsilon_{2} ; \varphi, t\right],
$$

where $\mathfrak{F}[\varepsilon ; \varphi ; t]$ is an abbreviation for either $(2.11)$ or $(2.12)$.

We first show that $\widetilde{F}$ has the stated tensorial properties. Letting $X$ denote a Cartesian frame of reference, then the constitutive equation is written in the $X$ frame as

$$
\sigma_{i j}^{X}=\widetilde{\mho}_{i j k l}^{X}\left[\varepsilon_{k l l}^{X} ; \varphi ; t\right],
$$

where the repeated indices imply the usual summation convention. In a second Cartesian reference frame $X^{\prime}$, the constitutive equation is written

$$
\sigma_{m n}^{X^{\prime}}=\mathfrak{F}_{m n p q}^{X^{\prime}}\left[\varepsilon_{p q}^{X^{\prime}}, \varphi, t\right]
$$

Thus, corresponding to each strain history component $\varepsilon_{k l}$ there is a functional $\mathfrak{F}_{i j k l}[, \varphi, t]$. Each functional $\mathfrak{F}_{i j k l}[, \varphi, t]$ will now be expressed in terms of the functionals $\mathfrak{F}_{m n p q}^{X^{\prime}}[, \varphi, t]$ by the transformation law of fourth order tensors.

Define $A$ to be the transformation that relates the $X$ frame and the $X^{\prime}$ frame. The transformation $A$ is represented through a set of time independent constants $a_{i j}$ such that

$$
x_{i}^{\prime}=a_{i j} x_{j}, \quad a_{i k} \alpha_{j k}=\delta_{i j} .
$$

Since $\sigma_{m n}^{X^{\prime}}$ and $\varepsilon_{p q}^{X^{\prime}}$ are second order tensors,

$$
\sigma_{i j}^{X}=a_{m i} a_{n j} \sigma_{m n}^{X^{\prime}} \quad \text { and } \quad \varepsilon_{p q}^{X^{\prime}}=a_{p k} a_{q l} \varepsilon_{k l l}^{X} .
$$

Combining Equations (3.3) and (3.4) yields

$$
\sigma_{i j}^{X}=a_{m n} a_{n i} \mathfrak{F}_{m n p q}^{X^{\prime}}\left[a_{p l} a_{q l} \varepsilon_{k l}^{X} ; \varphi ; t\right]
$$

Employ the linearity property of $\mathfrak{F}$ and compare Equation (3.6) to (3.2) to obtain

$$
\mathfrak{F}_{i j k l}^{X}\left[\varepsilon_{k l}^{X} ; \varphi ; t\right]=a_{m i} a_{n j} a_{p l} a_{q l} \mathfrak{F}_{m n p q}^{X^{\prime}}\left[\varepsilon_{k l l}^{X} ; \varphi ; t\right]
$$

Considering strain histories where all components are zero except $\varepsilon_{k l}^{X}=g$, then the equation above becomes

$$
\mathfrak{F}_{i j k l}^{X}[g ; \varphi ; t]=a_{m i} a_{n j} a_{p k} a_{q l} \mathfrak{F}_{m n p q}^{X^{\prime}}[g ; \varphi ; t]
$$

Thus the components of $\mathfrak{F}$ transform as a fourth order Cartesian tensor. For completeness, from the symmetry properties of $\sigma_{i j}$ and $\varepsilon_{i j}$, it also follows that

$$
\mathfrak{F}_{i j k l}=\mathfrak{\Im}_{j i k l}=\mathfrak{F}_{i j l k}=\mathfrak{F}_{j i l k} .
$$


Hence, the assertion of linearity allows the usual tensorial properties of a material response function to carry over to a functional type constitutive law.

It is possible to obtain an integral representation for the constitutive equations given in (2.11) and (2.12) if the functional $\mathfrak{F}$ is assumed to be linear in the strain history as defined in Assumption 2 and have the continuity properties defined in Definition 2.2 of Reference [1]. The continuity assumption essentially says that if two strain histories are close in value up to time $l$, then their corresponding stress states will be close at time $t$. The non-retroactivity property of Definition 2.2 is implied by the content of Assumption 1.

In stating the integral representation, it will be convenient to make use of an interval on the negative time axis. Let this interval be $(-\infty, 0)$. The strain histories are then assumed to belong to the class of functions which are continuous on $(-\infty, \infty)$ and zero on $(-\infty, 0)$. Recalling Assumption 1, it follows that $\sigma=0$ on $(-\infty, 0)$.

KöNIG and Metxner [7] consider a similar functional having all of the above mentioned properties along with that of translation invariance. They prove that the linear hereditary law can be expressed as a Riemann-Stieltjes integral whose integrator is uniquely determined from the functional $\mathfrak{F}$ and is independent of the strain history.

It will be useful to review the essential details of their proof. For each fixed time $t$ each component $\widetilde{F}_{i j k l}$ of $\widetilde{F}$ in (2.11) represents a linear functional on the class of scalar functions of form $\varepsilon(t-s)$, for $s$ in the interval $[0, t]$. Since it was assumed $\varepsilon(0)=\mathbf{0}$, this class of functions defines a linear space of functions $f(s)$ which are continuous on $[0, t]$ and have the property that $f(t)=0$. The HahnBanach Lemma [8], p. 114, allows extension of the functional to the linear space of functions which are continuous on $[0, t]$ with $f(t) \neq 0$. Then the Riesz Theorem [8], p. 100, can be applied to represent the functional as a Riemann-Stieltjes integral with a generating function $\hat{G}(\tau, t)$ where $t$ denotes the interval $[0, t]$ and $\tau$ is the integration variable in $[0, t]$. At this point in the proof the translation invariance condition for non-aging materials can be invoked to remove the dependence of the generating function on interval size.

For fixed $t$, the generating function $\hat{G}(\hat{\tau}, t)$ evaluated at some $\hat{\tau}$ in $[0, t]$ is defined as the value of the functional corresponding to the characteristic function for the sub-interval $[0, \hat{\imath}]$, which can be expressed in terms of the unit step function by $1(\hat{\tau}-s)$ for $s$ in $[0, t]$. It is important to note that this characteristic function corresponds to a strain history $\varepsilon(\tau)=1[\tau-(t-\hat{\tau})]$ which implies that $\hat{G}(\hat{\tau}, t)$ has a well defined physical interpretation. This interpretation will be considered in the next section.

The work of KöNIG and MEIXNER can be generalized to include the environmental history by allowing all possible strain histories for each fixed environmental history. Thus, employing the intermediate result of KöNIG and MerXNER preceding the application of the translation-invariance condition, we infer the following representation of a linear aging environmental dependent stress-strain law.

Let the stress history be related to the strain history by (2.11) where:

(a) $\varepsilon$ is defined and continuous on $(-\infty, \infty)$;

(b) $\varepsilon$ vanishes on $(-\infty, 0)$; 
and, the functional $\mathfrak{F}$ has the properties of linearity and continuity. Then Equation (2.11) can be written as

$$
\sigma_{i j}(t)=\int_{\mathbf{0}-}^{t} \varepsilon_{k l}(t-\tau) d_{t} \hat{G}_{i j k l}\left[\tau, t ; \varphi\left(\begin{array}{c}
t \underset{s=0}{t} s) \\
s=0
\end{array}\right]\right.
$$

where $d_{t} \hat{\boldsymbol{G}}$ denotes the differential with respect to $\tau$ for fixed $t$, and where $\hat{\boldsymbol{G}}$ has the following properties:

(c) $\hat{G}$ is defined for all $t$ in $(-\infty, \infty)$ and $\tau$ in $(-\infty, \infty) ; \hat{G}(\tau, t ; \varphi)=\mathbf{0}$ for $t<0$;

(d) for each fixed $t>0, \hat{\boldsymbol{G}}(\tau, t ; \varphi)=0$ for $\tau$ in $(-\infty, 0)$; and $(t, \infty), \hat{\boldsymbol{G}}$ may be non-zero only for $\tau$ in $[0, t]$;

(e) for each fixed $t, \hat{\boldsymbol{G}}$ is continuous on the right and of bounded variation for $\tau$ in $[0, t]$;

(f) $\hat{G}$ is a fourth order tensor whose components with respect to a Cartesian coordinate system satisfy

$$
\hat{G}_{i j k l}=\hat{G}_{j i k i}=\hat{G}_{i j l k}
$$

for $\tau$ and $t$ in the domain of definition.

Properties (c), (d), and (e) follow from the results of König and Meixner with the understanding that $\hat{G}$ is defined as zero before the beginning of the aging process (for negative $t$ ). The proof of $(f)$ is a consequence of Equation (3.8) and (3.9) and a similar result in [1, Theorem 2.5.].

The results in [7] assert left hand continuity. However, GURTIN and STERNBERG have observed [1, p. 306] that a trivial modification of the underlaying proof permits the assertion of right hand continuity which is more convenient for purposes considered here.

The stress-strain law (3.10) can further be specialized by considering the isotropy properties of the material in question. For the full isotropy group the fourth order tensor $\hat{G}$ can be expressed in terms of two scalar functionals (see, for example, Eqn. (2.34) of [9]). For the case of non-aging environmental independent linear viscoelastic materials, Rogkrs and PIPKIN [10], established tensorial representations of $\hat{G}$ for many of the sub-groups of the full isotropy group. The extension of these results to include Equation (3.10) follows directly if the isotropy transformations are valid for each environmental bistory and are assumed to be independent of the age of the material.

For convenience, assume the material is isotropic and homogeneous. Define the deviatoric stress and strain tensors as

and

$$
s_{i j}=\sigma_{i j}-1 / 3 \sigma_{k k} \delta_{i j}
$$

$$
e_{i j}=\varepsilon_{i j}-1 / 3 \varepsilon_{k k} \delta_{i j}
$$

where the quantity $\delta_{i j}$ is the Kronecker delta. GURTIN and STERn BerG's derivation of the representation of linear isotropic hereditary stress-strain laws [1, Theorem 
2.5] can now be applied to give

$$
s_{i j}(t)=\int_{0-}^{t} e_{i j}(t-\tau) d_{t} \hat{G}_{1}\left[\tau, t ; \varphi\left(\begin{array}{c}
t \\
s=0
\end{array}\right]\right.
$$

and

$$
\sigma_{k k}=\int_{0-}^{t} \varepsilon_{k k}(t-\tau) d_{t} \hat{G}_{2}\left[\tau, t ; \phi\left(\begin{array}{c}
t \underset{s=0}{t} s) \\
s=0
\end{array}\right.\right.
$$

for some fixed $t$. The function $\hat{G}_{1}$ is the shear relaxation functional and $\hat{G}_{2}$ the dilatational relaxation functional.

It is possible to remove the necessity of continuous stress and strain histories. In their study of non-aging environmental independent viscoelastic materials, Gurtin and STERnBerg treated discontinuous stress and strain histories as the limits of appropriate sequences of continuous stress and strain histories [1, Theorem 3.1]. This puts the concept of discontinuous stress and strain histories on a sound theoretical basis which leads to a natural physical meaning of the integral law. Application of their theorem in the case of aging materials is valid since the proof of the theorem does not depend on the size of the time interval $[0, t]$ and requires that $G_{i j k l}$ have a continuous first derivative on $[0, t]$; we assume this latter property.

According to property (d) following (3.10), $\hat{G}$ may have a jump discontinuity at $\tau=0$. By utilizing the theorem on step function integrators and the theorem on reduction of Stieltjes to Riemann integrals [11, Theorems 9.8, 9.9], the constitutive equation in (3.12) can be expanded as follows

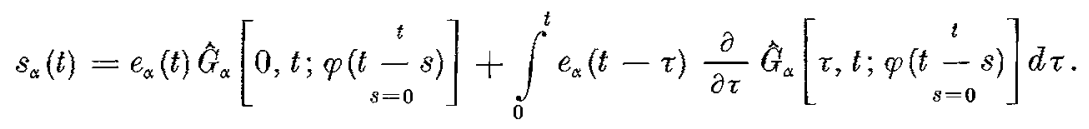

$s_{1}, e_{1}$ are corresponding deviatoric components defined in (3.11) and $\hat{G}_{1}$ is the shear relaxation functional. $s_{2}, e_{2}$ are the isotropic stress and strain components and $\hat{G}_{2}$ is the dilatational relaxation functional. An alternate form can be derived by integrating (3.13) by parts and changing variables to arrive at

$$
s_{\alpha}(t)=e_{\alpha}(0) \hat{G}_{\alpha}[t, t ; \varphi(t \underset{s=0}{t}-s)]+\int_{0}^{t} \hat{G}_{\alpha}\left[t-\xi, \varphi\left(\begin{array}{c}
t \underset{s=0}{t}-s) \\
-t
\end{array}\right] \frac{\partial e_{\alpha}(\xi)}{\partial \xi} d \xi .\right.
$$

In the preceding discussion it was assumed that the stress is determined by the strain and environmental histories. This assumption can be reversed, and one could assume the strain is determined by the stress and environmental histories. This amounts to interchanging the roles of $\sigma$ and $\varepsilon$ in the preceding development. The counterpart of the relaxation law given in Equation (3.10) is the creep law

$$
\varepsilon_{i j}(t)=\int_{0-}^{t} \sigma_{k l}(t-\tau) d_{t} \hat{J}_{i j k l}[\tau, t ; \varphi(t \underset{s=0}{t}-s)]
$$


For an isotropic material the aging hereditary creep law is

$$
\left.e_{\alpha}(t)=s_{\alpha}(t) \hat{J}_{\alpha}[0, t ; \varphi \underset{s=0}{\stackrel{t}{-}-s)}]+\int_{0}^{t} s_{\alpha}(t-\tau) \frac{\partial}{\partial \tau} \hat{J}_{\alpha}[\tau, t ; \underset{s=0}{t} \underset{s}{-} s)\right] d \tau
$$

where $\hat{J}_{1}$ and $\hat{J}_{2}$ are the creep functionals in shear and dilatation respectively.

The derivation of the integral representation for the functional in (2.12) differs slightly from that for (2.11). For each fixed $t$ each component $\mathfrak{F}_{i j k l}$ of $\mathfrak{F}$ in (2.12) represents a linear functional on the linear space of scalar functions $f(s)$ which are continuous on $[0, t]$ and have the property $f(0)=0$. As before, the Hahn-Banach Lemma and the Riesz Theorem are applied to represent the functional as a Riemann-Stieltjes integral. However, in this case, with its physical interpretation in mind, the generating function of the Stieltjes integral $G^{*}(\tau, l)$ is now defined as the value of the functional corresponding to the characteristic function for the sub-interval $[\tau, t]$ of the interval $[0, t]$. This characteristic function coincides with the argument of the linear functional for the strain history $\varepsilon(s)=1(s-\tau)$. The proof of the Riesz Theorem is unaffected by this change in definition of the generating function. The properties of $G^{*}(\tau, t)$ differ slightly from those of the earlier proof and are summarized below.

For the linear aging environmental dependent stress-strain law denoted by (2.12) having the properties of linearity and continuity, the stress history can be expressed in terms of the strain history through the relation

$$
\sigma_{i j}(t)=-\int_{0}^{t+} \varepsilon_{k l}(\tau) d_{t} G_{i j k l}^{*}[\tau, t ; \underset{s=0}{t}]
$$

where $d_{t} G^{*}$ denotes the differential with respect to $\tau$ for fixed $t$ and where $G^{*}$ has properties (c), (d) and (f) mentioned after (3.10). The change in definition of the generating function gives rise to the minus sign in (3.17) and changes property (e) to assert left-hand continuity.

The remarks made in regard to (3.10) also apply to (3.17). Confining our attention to isotropic materials, it is sufficient to consider scalar versions of (3.17). In view of the remarks following (3.14), the strain histories in (3.17) may now have jump discontinuities.

According to its property (d), $G^{*}$ may have a jump discontinuity at $\tau=t$. Assuming that $G^{*}(\tau, t ; \varphi)$ has a continuous first derivative for $\tau$ in $(0, t]$, the methods leading to (3.13) also can be used to reduce (3.17) to

$$
\sigma(t)=\varepsilon(t) G^{*}(t, t ; \varphi)-\int_{0}^{t} \varepsilon(\tau) \frac{\partial}{\partial \tau} G^{*}(\tau, t ; \varphi) d \tau
$$

An alternate form for (3.17) can be obtained by integrating (3.18) by parts to give

$$
\sigma(t)=\varepsilon(0) G^{*}(0, t ; \varphi)+\int_{0}^{t} G^{*}(\tau, t ; \varphi) \frac{\partial}{\partial \tau} \varepsilon(\tau) d \tau
$$


Finally, the creep law counterpart of the relaxation law (3.17) is given by

$$
\varepsilon(t)=-\int_{0}^{t} \sigma(\tau) d_{t} J^{*}(\tau, t ; \underset{\substack{t \\ s=0}}{s})
$$

where $J^{*}$ has the same properties as $G^{*}$.

\section{Properties of the Model}

To investigate the physical meaning of the variables in the generating functionals of Equations (3.10) and (3.17), let the strain history be given by

$$
\varepsilon(t)=1(t-a) .
$$

Using Equation (4.1) to evaluate the scalar equivalent of (3.10) gives

$$
\begin{array}{ll}
\sigma(t)=\hat{G}[t-a, t ; \varphi(t \underset{s=0}{t}-s)], & t \geq a, \\
\sigma(t)=0, & t<a .
\end{array}
$$

Thus, $\hat{G}\left[t-a, t ; \varphi\left(\begin{array}{c}t \\ \stackrel{t}{-}-s)\end{array}\right]\right.$ is the stress at the present time $t$ due to a unit step strain that was applied at time $a$. The quantity $(t-a)$ represents the time that has elapsed since the strain was applied. The relaxation functional $\hat{G}$ can be interpreted as a surface for each environmental history. A typical surface is shown in Fig. 1.

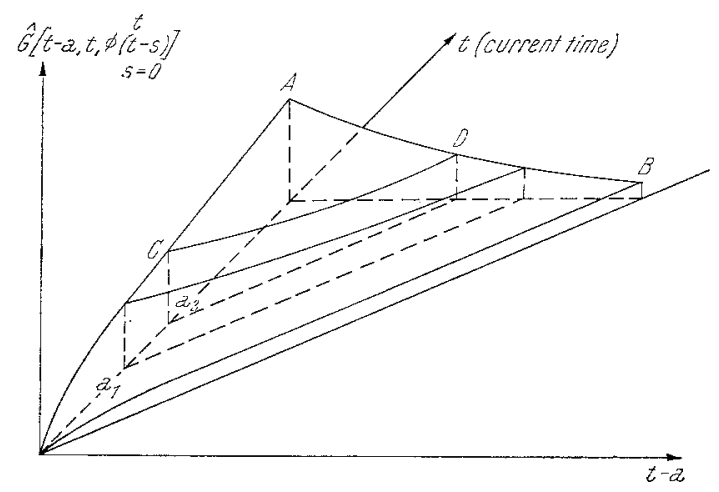

Fig. 1. A typical relaxation surface $\hat{G}$ for the response function given by equation (4.2)

Using (4.1) to evaluate the scalar equivalent of (3.16) gives

$$
\begin{array}{ll}
\sigma(t)=G^{*}\left[\begin{array}{rr}
a, t ; \varphi(s) \\
s=0
\end{array}\right], & t \geq a, \\
\sigma(t)=0, & t<a .
\end{array}
$$


Although (4.3) has the same physical interpretation as (4.2), the argument $\underline{a}$ denotes the actual time that the unit step strain was applied. A typical relaxation surface corresponding to $G^{*}(\tau, t ; \varphi)$ is shown in Fig. 2.

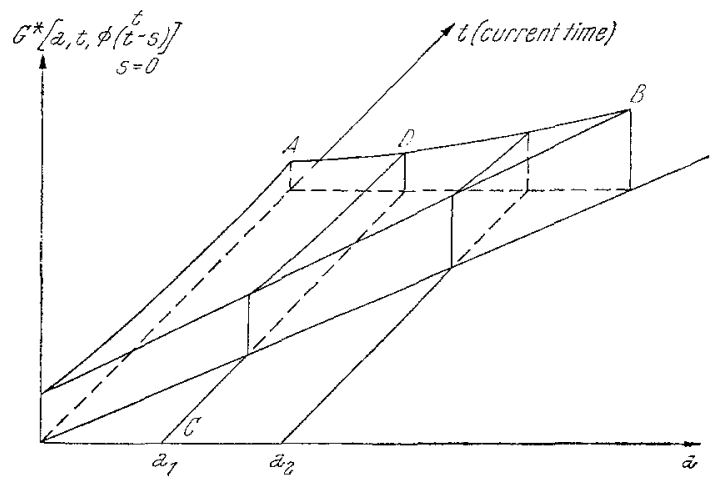

Fig. 2. A typical relaxation surface $G^{*}$ for the response function given in equation (4.3)

In Figures 1 and 2 the curve $A B$ represents the value of the stress ( $\hat{G}$ or $G^{*}$ respectively) at time $t$ due to a unit step strain applied at values of $\underline{a}$. The curves $C D$ in Figures 1 and 2 represent the stress at different times $t$ due to the $a$ unit step strain applied at time $a$. Utilizing the physical interpretation given for $\hat{G}$ in (4.2) and $G^{*}$ in (4.3), integrals $(3.13),(3.14),(3.18)$ and (3.19) can be constructed by superposition arguments of the type given in FLÜGGE [12]. Equations (3.13) and (3.18) arise by considering the strain history to be approximated by a series of strain pulses. Equations (3.14) and (3.19) arise by considering the strain history to be the limit of a series of step strains. Recalling that the first argument in $\hat{G}$ represents time elapsed since a step strain, the first term in (3.13) implies that $G[0, t ; p]$ is the instantaneous elastic response due to a unit strain impulse applied at time $t$. Similarly, $G^{*}[0, t ; \varphi]$ in $(3.18)$ also represents an instantaneous elastic response.

At this point it is easy to introduce the relationships between the creep and relaxation functions for aging materials. The relaxation functional in (4.2) may be interpreted as the stress history required to produce the unit-step strain history $1(t-\alpha)$. Thus, substituting (4.1) and (4.2) into the scalar version of (3.15) yields

$$
\begin{aligned}
1(t-a) & =\int_{0-}^{t} \hat{G}[t-\tau-a, t-\tau] d_{t} \hat{J}(\tau, t) \\
& =\int_{0-}^{t} \hat{J}[t-\xi, t] d_{\alpha} \hat{G}[\xi-a, \xi],
\end{aligned}
$$

where the last line follows by integrating by parts and changing variables. Two additional relationships may be calculated from (3.10) when $\sigma(t)=\mathbf{1}(t-a)$ and the resulting creep response is $\varepsilon(t)=\hat{J}(t-a, t)$ calculated from (3.15). These

${ }^{1}$ For convenience in writing, dependence on $\varphi$ is suppressed. 
relations can be formally obtained interchanging $\hat{J}$ and $\hat{G}$ in (4.4). They represent four equivalent forms of a Volterra integral equation of the second kind which may be solved for one of the material response functions when the other is determined experimentally. If, say, $\hat{J}$ is known, the most useful form for finding $\hat{G}$ appears to be that obtained by reducing the first line of (4.4) to the form

$$
\hat{G}[t-a, t] \hat{J}[0, t]+\int_{a}^{t} \hat{G}(\xi-a, \xi) \frac{\partial}{\partial(t-\xi)} \hat{J}(t-\xi, t) d \xi=1, t \geq a .
$$

Obtaining (4.5) required use of a jump discontinuity in $\hat{J}$ at $\tau=0$, the vanishing of $\hat{G}$ for $\tau>t-a$ and the change of variables $\tau=t-\xi$. This appears to be the only form in which given the constant $a, G(\xi-a, \xi)=f(\xi)$, is a function of only one variable. This solution represents the curve $C D$ shown in Fig. 1.

Analogous relations for the second form of stress-strain relation can be derived by using $\varepsilon(t)=1(t-a)$ and stress response (4.3) in (3.20) to yield

$$
1(t-a)=-\int_{0}^{t+} G^{*}[a, \tau] d_{t} J^{*}[\tau, t]
$$

Using $\sigma=1(t-a)$ and corresponding strain $J^{*}(a, t)$ in (3.17) yields

$$
1(t-a)=-\int_{0}^{t *} J^{*}(a, \tau) d_{t} G^{*}(\tau, t) .
$$

If $J^{*}$ is known, $G^{*}$ for fixed $a$ can be found from the Volterra integral equation (4.6) which when reduced to a Riemann integral has the form

$$
G^{*}[a, t] J^{*}(t, t)-\int_{a}^{t} G^{*}(a, \tau) \frac{\partial}{\partial \tau} J^{*}(\tau, t) d \tau=1, \quad t \geq a .
$$

Although the integral representations (3.10) and (3.17) show that the relaxation functions depend on environmental history, very little can be said about the explicit nature of this dependence. Including the environmental history on the interval $[0, t]$ in (4.2) and (4.3) implies that the stress, and consequently the material properties, depend on the interaction of the aging phenomenon and the environment at all times since the material was made. ${ }^{2}$

In a general discussion of concrete as an aging material, SAcKmaN [5] pointed out that an explicit representation for the dependence of relaxation functional (4.2) or (4.3) on the environmental history $\varphi$ might be derivable using the corresponding theory for non-aging materials as a guide. Such an approach requires that creep or relaxation measurements be made at steady environments. For example, [5] contains creep curves measured at various constant temperatures and humidity levels. SACKMAN considered the possibility that the curves for, say,

2 A discussion of environmental effects in non-aging materials [3] utilizes the apparently reasonable assumption that for a strain history of form (4.1), $\hat{G}$ depends on the history of $\varphi$ only during straining, i.e., on the interval $[a, t]$. 
various temperatures, could be related by translations along a logarithmic time axis, as is possible for non-aging materials. The data also suggests the possibility that these families of creep curves can be related by vertical scaling. The creep function for transient environments can then be derived using relations for steady environments. A general study for non-aging materials in which creep functions are related by both vertical scaling and shifts along the log time axis has recently been given in [3].

In conclusion, we briefly discuss the relative merits of the stress-strain law in forms (3.10) and (3.17). Note that the law in form (3.10) can be transformed into form (3.17) by letting $t-\tau=\tau^{\prime}$ and by equating (4.2) and (4.3), which are different expressions for the stress relaxation history arising from the same step strain history. This is to be expected because the above transformation causes events measured with respect to current time $t$ in (3.10) now to be measured with respect to creation time as in (3.17). Furthermore, curve $C D$ in Fig. 1 is mapped into $C D$ in Fig. 2.

As $t$ becomes large, it is reasonable to expect the "aging" laws (3.10) and (3.12) to approach the standard integral non-aging law of linear viscoelasticity. For steady environments, (3.10) approaches this law if $\hat{G}(\tau, t) \rightarrow \hat{G}(\tau)$. For (3.17), however, $G^{*}(\tau, t) \rightarrow \hat{G}(t-\tau)$, and then a change of variables is necessary to get the proper form. A discussion for general environments is presented in Section 5 .

Since (3.10) can be transformed into (3.17), or vice-versa, a preference for one form or the other should be based on analytical convenience. SAcKmaN [5] has pointed out that (3.17) has been more convenient to work with. Furthermore, ARUTYUNYan [4] has made (3.17) the basis for his study of the mechanies of concrete. In the non-aging theory, manipulations of the integral operator of the stress strain law can be expressed in terms of an operator algebra which has been invaluable in solving problems. Although an operator algebra can be developed for both forms of the law, as is shown in Section $6,(3.12)$ appears to be preferable because of economy in writing.

\section{Specialization for Non-Aging Materials}

Although the constitutive equations (2.11) and (2.12) are intended as the starting point for a discussion of an aging environmental-dependent viscoelastic material, they can also be applied to non-aging materials. Thus, instead of making a specific constitutive assumption for a theory in which aging effects are absent, it is demonstrated that under appropriate conditions, as $t$ becomes large, (2.11) and (2.12) approach a constitutive equation describing a non-aging material. ${ }^{3}$ The environmental history experienced by a particle while aging interacts with the aging process to produce a fully cured (non-aging) material with certain mechanieal properties.

In considering this transition in more detail, let $T$ be a measure of the time required for the material to become fully cured ( $T$ may depend on the inter-

3 This idea was incorporated by ARUTrunyan ([4], p. 29) in suggesting analytic expression for the relaxation function $G^{*}(\tau, t)$ in $(3.17)$. 
action). Assume that $\varepsilon=0$ on $[0, T]$ so that there are no initial stresses in the cured state. Then $\sigma(t)$ in the cured state, i.e. for $t>T$, depends explicitly on the strain history $\varepsilon(\tau)$ and the environmental history $\varphi(\tau)$ only on the interval $[T, t]$. If $\mathfrak{F}$ denotes a new functional, then (2.11) and (2.12) reduce to

$$
\sigma(t)=\widetilde{F}\left[\begin{array}{cc}
t & t \\
\varepsilon\left(\tau_{1}\right) & \varphi\left(\tau_{2}\right) ; t \\
\tau_{1}=T & \tau_{2}=T
\end{array}\right]
$$

and

$$
\sigma(t)=\mathfrak{F}\left[\begin{array}{cc}
t-T & t-T \\
\varepsilon\left(t-s_{1}\right) & i p\left(t \underset{s}{-} s_{2}\right) ; t \\
s_{1}=t_{0} & s_{2}=t_{0}
\end{array}\right]
$$

for $t \gg T$. The new functional $\mathfrak{F}$ describes the mechanical properties of the fully cured material, which may be different for each $\varphi(t)$ on $[0, T]$.

The constitutive assumption, as given in (2.1), prescribes the physical quantities which can affect the stress history. We next make an assumption on the nature of this dependence in the cured state.

Assumption 3. Let $\varepsilon=\mathbf{0}$ on $\left[0, t_{0}\right]$ for $t_{0}>T$. Then the state of stress at any $t \geq t_{0}$ is independent of the environmental history on $\left[T, t_{0}\right]$.

In more physical terms, the environmental history when the cured material is "on the shelf" does not affect later mechanical response. Letting $\mathfrak{F}$ denote another new response functional, the constitutive equations (5.1) or (5.2) for strain histories with $\varepsilon=\mathbf{0}$ on $\left[0, t_{0}\right]$ can be rewritten as

$$
\sigma(t)=\widetilde{F}\left[\begin{array}{cc}
t & t \\
\varepsilon\left(\tau_{1}\right) ; & \varphi\left(\tau_{2}\right) ; t \\
\tau_{1}=0 & \tau_{2}=0
\end{array}\right]
$$

or

$$
\sigma(t)=\widetilde{F}\left[\begin{array}{cc}
t-t_{0} & t-t_{0} \\
\varepsilon\left(t-s_{1}\right) & p\left(t-s_{2}\right) \\
s_{1}=0 & s_{2}=0
\end{array}\right]
$$

for $t>t_{0}>T>0$.

Selecting $t_{0}$ as the new origin for convenience, the constitutive equations (5.3) and (5.4) assume the form of (2.11) and (2.12). In the sequel, $\varepsilon(t)$ is assumed to vanish for $t<0$ and is continuous on $[0, \infty)$. Assume $\varphi(t)$ is continuous for all times since the curing time.

With the establishment of the above notions, non-aging response can now be characterized. A material is said to be non-aging if the constitutive equation is such that the mechanical response is unaltered by a shift of the strain and concurrent environmental histories with respect to, say, the curing time $T$.

Given a set of strain and environmental histories $\varepsilon$ and $\varphi$ satisfying the above mentioned conditions, define a second set by the relations

and

$$
\hat{\boldsymbol{\varepsilon}}(\tau)=\varepsilon(\tau+a) \quad \text { for all } \tau \text { since the cure time, }
$$

$$
\begin{array}{ll}
\hat{\varphi}(\tau)=\varphi(\tau+a) & \tau \text { on }[-a, \infty) \\
\hat{\varphi}(\tau) \ldots \text { arbitrary } & \text { from cure time to } \tau=-a,
\end{array}
$$


where " $a$ " is an arbitrary constant. The stress state $\hat{\sigma}$ which results at $t-a$ due to strain history $\hat{\varepsilon}$ and environmental history $\hat{\varphi}$ is given by, say, (5.3) as

$$
\hat{\sigma}(t-a)=\mathfrak{\mho}\left[\begin{array}{cc}
t-a & t-a \\
\hat{\varepsilon}\left(\tau_{1}\right) ; & \hat{\varphi}\left(\tau_{2}\right) ; t-a \\
\tau_{1}=-a & \tau_{2}=-a
\end{array}\right] .
$$

According to the above definition, the material is said to be non-aging if the stress states which result from equations (5.3) and (5.6) are identical; i.e., $\hat{\sigma}(t-a)$. $=\sigma(t)$. The constitutive equation is said to be translation invariant (see [1]). This condition together with (5.5), leads to the following restriction on $\mathfrak{F}$ :

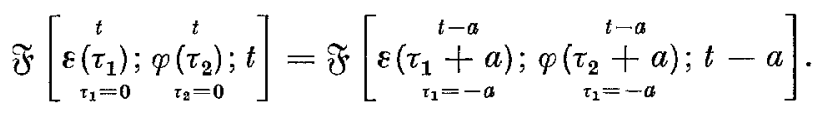

Letting $a=t-\xi, \xi$ being an arbitrary constant, and defining $\tau_{n}-\xi=-s_{n}$, $n=1,2$, Equation (5.7) can be written as

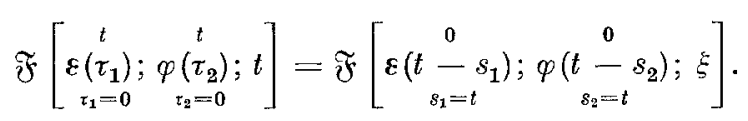

Since $\xi$ is arbitrary, $\mathfrak{F}$ cannot depend explicitly on time $t$. Thus, for translation invariant, environmental dependent materials, the viscoelastic response can be characterized by the functional relation

$$
\sigma(t)=\mathscr{F}\left[\begin{array}{cc}
t \\
\varepsilon\left(t \underset{t}{t} s_{1}\right) \\
s_{1}=0
\end{array} \underset{\substack{t \\
s_{2}=0}}{\left.-s_{2}\right)}\right]
$$

where $\mathfrak{F}$ is again defined as a new functional.

A similar result can be obtained for the constitutive equation in form (5.4). The stress $\hat{\sigma}$ at time $t-a$ is given by

$$
\hat{\boldsymbol{\sigma}}(t-a)=\widetilde{\mho}\left[\hat{\boldsymbol{\varepsilon}}\left(t-\stackrel{t}{a}-s_{1}\right) ; \hat{\phi}\left(t-\stackrel{t}{a}-s_{s_{2}=0}\right) ; t-a\right] .
$$

Employing the translation invariance principle, $\hat{\sigma}(t-a)=\sigma(t)$ and (5.5) gives

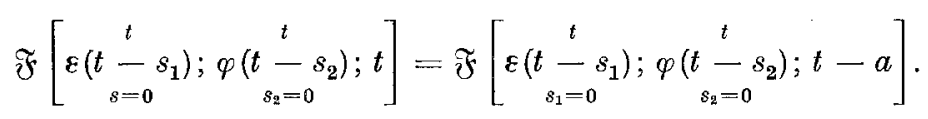

Thus it can be seen that the constitutive functional $\tilde{F}$ is independent of the explicit time variable $t$ since the constant $\underline{a}$ is arbitrary. The constitutive equation as implied by (5.9) is identical to (5.8).

The resultant stress-strain law for a non-aging material, Equation (5.8), is the usual starting point for an analysis involving only environmental effects. Many such studies have been carried out for both temporally constant and transient environments. Some examples of this work are given in References [2], [3], and [13]. 


\section{Appendix}

\section{Operator Algebra}

The constitutive equations in form (3.12) or (3.17) can be conveniently represented using an operator notation. Operations involving (3.12) or (3.17) form an operator algebra which facilitates their manipulations in solving boundary value problems.

Definition 1. Let the scalar function $f(t)$ be defined and have the following properties on $(-\infty, \infty)$ :

(a) $f(t) \quad$ vanishes on $(-\infty, 0)$;

(b) $f(t)$ is continuous on the right in the interval $[0, \infty)$;

(c) $\frac{d f}{d t} \quad$ exists and is continuous on $[0, \infty)$.

Definition 2. Let the scalar function $\psi(\tau, t)$ have the following properties:

(a) $\psi(\tau, t)$ is defined for all $t$ and $\tau$ in $(-\infty, \infty) ; \psi(\tau, t)=0$ for $t<0$.

(b) For each fixed $t \geq 0, \psi(\tau, t)=0$ for $\tau$ in $(-\infty, 0)$ and $(t, \infty), \psi(\tau, t)$ may be non-zero for $\tau$ in $[0, \mathrm{t}]$.

(c) $\frac{\partial \psi}{\partial \tau}(\tau, t)$ exists and is continuous with respect to $\tau$ on $[0, t]$.

In the remainder of this section, all functions will be assumed to have these properties unless otherwise stated. These properties are sufficient to guarantee the existence of the Stieltjes integrals to be used (see [11], Theorem 9-26).

Functions of one time variable $f(t)$ will usually arise from operations performed on stress or strain. Functions of two time variables $\varphi(\tau, t)$ will usually represent material response functions or operations involving them.

Definition 3. If $\tau$ is fixed in $\psi(\tau, t)$, the resulting function of $t$ is denoted by

$$
{ }_{\tau} \psi(t)=\psi(\tau, t) \text {; }
$$

if $t$ is fixed in $\psi(\tau, t)$, the resultant function of $\tau$ is denoted by

$$
\psi_{t}(\tau)=\psi(\tau, t)
$$

if $s$ is fixed in $\varphi(t-s, l)$, the resultant function of $t$ is denoted by

$$
\Phi(s, t)={ }_{s} \Phi(s, t)=\varphi(t-s, t) .
$$

Note that $\Phi$ has properties (a) and (b) of Definition 2.

Definition 4. The Riemann Stieltjes integrals

and

$$
I_{1}(t)=\int_{0-}^{t} f(t-\tau) d_{t} \psi(\tau, t)=\int_{0-}^{t} f(t-\tau) d \psi_{t}(\tau),
$$

$$
I_{2}(t)=\int_{0-}^{t} \psi(t-\tau, t) d f(\tau)=\int_{0-}^{t} \psi_{t}(t-\tau) d f(\tau)
$$


will be denoted respectively by

$$
I_{1}=f * d \psi_{t} \quad \text { and } \quad I_{1}=\psi_{t} * d f .
$$

The Riemann-Stieltjes integrals

and

$$
X_{1}(s, t)=\int_{0-}^{t} \varphi(t-\tau-s, t-\tau) d_{t} \psi(\tau, t)=\int_{0-}^{t} \Phi(s, t-\tau) d \psi_{t}(\tau)
$$

$$
X_{2}(s, t)=\int_{0-}^{t} \psi(t-\tau, t) d_{s} \varphi(\tau-s, \tau)=\int_{0-}^{t} \psi_{t}(t-\tau) d_{s} \Phi(s, \tau)
$$

will be denoted respectively by

$$
X_{1}={ }_{s} \Phi * d \psi_{t}, \quad X_{2}=\psi_{t} * d\left(_{s} \Phi\right)
$$

These integrals are similar in structure to the Stieltjes convolutions discussed in [1] and hence are given similar notation. This notation, although perhaps cumbersome, will be useful in representing the algebraic like manipulations of $(6.2 \mathrm{a})$ and (6.3a).

These manipulations and their proofs are analogous to those given in [1].

$$
\text { Properties of }(6.2 \mathrm{a}) \text { and }(6.3 \mathrm{a})
$$

(a) $f * d \psi_{t}$ and $\psi_{t} * d f$ are defined for $t$ on $(-\infty, \infty)$ and vanish on $(-\infty, 0)$; $\varphi_{s} * d \psi_{t}$ and $\psi_{t} * d \varphi_{s}$ vanish for $t<0$; for fixed $t \geq 0$, they vanish for $s$ in $(-\infty, 0)$ and in $(t, \infty)$.

(b) $f * d \psi_{t}=\psi_{t} * d f, \quad\left({ }_{s} \Phi\right) * d \psi_{t}=\psi_{t} * d\left({ }_{s} \Phi\right)$.

(c) $\psi_{t} * d\left[\Phi_{i} * d f\right]=\left[\psi_{t} * d\left({ }_{\delta} \Phi\right)\right] * d f$.

(d) $f * d \psi_{t}$ is linear in $f$ and $\psi$.

(e) $f * d \psi_{t}=0$ implies $f=0$.

(f) if $f=1(t-s)$, then $\psi_{t} * d f=\psi(t-s, t)$.

(g) $f * d \psi_{t}=\psi(0, t) f(t)+\int_{0}^{t} f(t-\tau) \frac{\partial \psi}{\partial \tau} d \tau$

$$
\begin{aligned}
& \psi_{t} * d f=f(0) \psi(t, t)+\int_{0}^{t} \psi_{t}(t-\tau) \frac{\partial f}{\partial \tau} d \tau \\
& \left({ }_{s} \Phi\right) * d \psi_{t}=\psi(t-s, t) \psi(0, t)+\int_{s}^{t-s} \varphi(t-s-\tau, t-\tau) \frac{\partial}{\partial \tau} \psi(\tau, t) d \tau \\
& \psi_{t} * d\left({ }_{s} \Phi\right)=\psi(t-s, t) \varphi(0, s)+\int_{s}^{t} \psi(t-\tau, t) \frac{\partial}{\partial \tau} \varphi(\tau-s, \tau) d \tau
\end{aligned}
$$

Proof. Properties (d), (f) follow directly from the properties of RiemannStieltjes integrals ([11], Theorem 9-3, 9-9, respectively). 
To establish the properties in (a), use the fact that the functions $\varphi$ and $\psi$ appearing in (6.2) and (6.3) have properties given in Definition 2.

The first operational property stated in (b) is analogous to property (b) of Theorem 1.2 in [1]. The proofs are identical, both resting on an application of the theorem of integration of Stieltjes integrals by parts [11, Theorem 9-6] for fixed $t$; followed by a change of variables and noting the property that $\psi(-\alpha, t)=0$. The proof of the second part of $(b)$ is similar. Integrating by parts, noting that $\Phi(s, 0)=0$ by property (b) of Definition 2 , and that $\psi(-\alpha, t)=0$ gives

$$
\int_{-\infty}^{t} \Phi(s, t-\tau) d \psi_{t}(\tau)=-\int_{-\infty}^{t} \psi_{t}(\tau) d_{t}\left[{ }_{s} \Phi(t-\tau)\right] .
$$

Let $t-\tau=\xi$ and use the theorem on change of variables in a Stieltjes integral $[11$, Theorem 9-7]. Then since $\Phi(s, \tau)=0$ for $\tau<s$ and $\psi(\tau, t)=0$ for $\tau>0$, (6.4) becomes

$$
\int_{-\infty}^{t} \Phi(s, t-\tau) d \psi_{t}(\tau)=\int_{-0}^{t} \psi_{t}(t-\tau) d\left[{ }_{s} \Phi(\tau)\right]
$$

which by (6.3) completes the proof.

To prove the first part of $(\mathrm{g})$, define $\hat{\psi}(\tau, t)$, continuous on $(-\infty, t]$, by

$$
\psi(\tau, t)=\hat{\psi}(\tau, t)+\psi(0, t) 1(\tau) .
$$

Substituting (6.5) in (6.2a), using property (d) and the theorem on step function integrators,

$$
\left(f * d \psi_{t}\right)(t)=\psi(0, t) f(t)+\int_{0}^{t} f(t-\tau) d \hat{\psi}_{t}(\tau) .
$$

Observing that for $\tau$ in $[0, t]$

$$
\frac{\partial \psi(\tau, t)}{\partial \tau}=\frac{\partial \hat{\psi}(\tau, t)}{\partial \tau}
$$

and using the theorem on reduction of Stieltjes integrals to Riemann integrals, (6.6) und (6.7) yields

$$
\left(f * d \psi_{t}\right)(t)=\psi(0, t) f(t)+\int_{0}^{t} f(t-\tau) \frac{\partial \psi(\tau, t)}{\partial \tau} d \tau
$$

as asserted. Interchanging $f$ and $\psi_{t}$ yields the second part of $(\mathrm{g})$. The third is obtained from the first two by letting $f(\tau)=\varphi(\tau-s, \tau)=\varphi(s, \tau)$, for fixed $s$, and then recalling that $\Phi(s, \tau)=0$ for $\tau<s$. To obtain the last part, let $\Phi(s, \tau)=\varphi(\tau-s, \tau)=\varphi(0, s) 1(\tau-s)+\widetilde{\varphi}(\tau-s, \tau)$, where $\tilde{\varphi}$ is continuous for $\tau$ in $(-\infty, \infty)$.

To verify (e), set $f * d \psi_{t}=0$ in the first part of $(\mathrm{g})$ and make the change variable $\xi=t-\tau$ to arrive at

$$
0=\psi(0, t) f(t)+\int_{0}^{t} f(\xi) \frac{\partial \psi(t-\xi, t)}{\partial(t-\xi)} d \xi .
$$

Thus, Equation (6.11) is a homogeneous Volterra integral equation of the second kind for $f(t)$, when $\psi(0, t) \neq 0$. The uniqueness properties of Volterra integral 
equations $[14, p .35]$ verify that $f(t)=0$ on $[0, t]$. For the case when $\psi(0, t)=0$ let

$$
g(t-\xi, t)=\frac{\partial \psi(t-\xi, t)}{\partial(t-\xi)}
$$

then differentiate Equation (6.8) with respect to $t$ to get

$$
0=g(0, t) f(t)+\int_{0}^{t} f(\xi) \frac{\partial g(t-\xi, t)}{\partial t} d \xi .
$$

Thus, once again it follows that $f(t)$ must equal zero.

To prove (c) let

$$
\left.\theta_{1}={ }_{s} \Phi * d \psi_{t}\right) * d f=\int_{-\alpha}^{t}\left\{\int_{-\alpha}^{t-s} \varphi(t-\tau-s, t-\tau) d_{t} \psi(\tau, t)\right\} d f(s) .
$$

Expanding the Stieltjes integrals in (6.9) according to relations developed in (g)

$$
\begin{aligned}
\theta_{1}= & \int_{-\alpha}^{t}\left\{\varphi(t-s, t) \psi(0, t)+\int_{0}^{t-s} \varphi(t-\tau-s, t-\tau) \frac{\partial \psi(\tau, t)}{\partial \tau} d \tau\right\} d f(s) \\
= & \psi(0, t) \int_{-\alpha}^{t} \varphi(t-s, t) d f(s)+f(0) \int_{0}^{t} \varphi(t-\tau, t-\tau) \frac{\partial \psi(\tau, t)}{\partial \tau} d \tau \\
& +\int_{0}^{t} \int_{0}^{t-s} \varphi(t-\tau-s, t-\tau) \frac{\partial \psi(\tau, t)}{\partial \tau} \frac{d f(s)}{d s} d \tau d s .
\end{aligned}
$$

Interchanging the order of integration, the double integral can be rewritten as

$$
\begin{aligned}
\theta_{1}= & \psi(0, t) \int_{-\infty}^{t} \varphi(t-s, t) d f(s) \\
& +\int_{0}^{t}\left\{\varphi(t-\tau, t-\tau) f(0)+\int_{0}^{t-\tau} \varphi(t-\tau-a, t-\tau) \frac{d f(s)}{d s} d s\right\} \frac{\partial \psi(\tau, t)}{\partial \tau} d \tau .
\end{aligned}
$$

Again utilizing $(\mathrm{g}), \theta_{1}$ can be written as Stieltjes integrals:

$$
\begin{aligned}
\theta_{1} & =\psi(0, t) \int_{-\alpha}^{t} \varphi(t-s, t) d f(s)+\int_{0}^{t}\left\{\int_{-\alpha}^{t-\tau} \varphi(t-\tau-s, t-\tau) d f(s)\right\} \frac{\partial \psi(\tau, t)}{\partial \tau} d \tau \\
& =\int_{-\alpha}^{t}\left\{\int_{-\alpha}^{t-\tau} \varphi(t-\tau-s, t-\tau) d f(s)\right\} d_{t} \psi(\tau, t) . \\
& =\int_{-\alpha}^{t}\left\{\int_{-\alpha}^{t} \varphi(s, t-\tau) d f(s)\right\} d_{t} \psi(\tau, t) .
\end{aligned}
$$

In terms of the notation defined in (6.2), (6.3), $\theta_{1}$ can be expressed by

$$
\left({ }_{s} \Phi * d \psi_{t}\right) * d f=\left(\varphi_{t} * d f\right) * d \psi_{t} .
$$


The desired result is then obtained using the commutativity properties (b).

Similar properties can be developed for the integral operator in (3.17). The most convenient notation for this operator appears to be that obtained by suppressing the independent variable of integration in the Riemann-Stieltjes integrals, i.e.

$$
\begin{aligned}
\int_{-0}^{t} \psi(\tau, t) d f(\tau) & =\int_{-0}^{t} \psi_{t} d f, \\
\int_{0}^{t+} \varphi(s, \tau) d_{\tau} \psi(\tau, t) & =\int_{0}^{t+} s \varphi d \psi_{t} .
\end{aligned}
$$

Properties of (6.14)

(a) $\int_{0}^{t+} f d \psi_{t}, \int_{0-}^{t} \psi_{t} d f$ are defined for $t$ in $(-\infty, \infty)$ and vanish on $(-\infty, 0)$.

$\int_{0}^{t+} s \varphi d \psi_{t}, \int_{0}^{t} \psi_{t} d\left({ }_{s} \varphi\right)$ vanish for $t<0$; for fixed $t \geq 0$, they vanish for $s$ in $(-\infty, 0)$ and $(t, \infty)$.

(b) $\int_{0}^{t+} f d \psi_{t}=-\int_{0-}^{t} \psi_{t} d f, \int_{0}^{t+} s_{s} \varphi d \psi_{t}=-\int_{0-}^{t} \psi_{t} d\left({ }_{s} \varphi\right)$

(c) $\int_{0-}^{t}\left[\int_{0-}^{t} \psi_{t} d\left({ }_{s} \varphi\right)\right] d f=\int_{0-}^{t} \psi_{t} d\left[\int_{0-}^{t} \varphi / d f\right]$

(d) $\int_{0}^{t+} f d \psi_{t}$ is linear in $f$ and $\psi_{t}$

(e) $\int_{0}^{t+} f d \psi_{t}=0$ implies $f(t)=0$ for all $t$

(f) if $f=1(t-s)$, then $\int_{0}^{t} \psi_{t} d f=\psi(s, t)$

(g) $\int_{0}^{t+} f d \psi_{t}=-f(t) \psi(t, t)+\int_{0}^{t} f(\tau) \frac{\partial \psi(\tau, t)}{\partial \tau} d \tau$

$\int_{0-}^{t} \psi_{t} d f=f(0) \psi(0, t)+\int_{0}^{t} \psi(\tau, t) \frac{\partial f(\tau)}{\partial \tau} d \tau$

$$
\begin{aligned}
& \int_{0}^{t+} s_{s} d \psi_{t}=-\varphi(s, t) \psi(t, t)+\int_{s}^{t} \varphi(s, \tau) \frac{\partial \psi(\tau, t)}{\partial \tau} d \tau \\
& \int_{-0}^{t} \psi_{t} d\left({ }_{s} \varphi\right)=\psi(s, t) \varphi(s, s)+\int_{s}^{t} \psi(\tau, t) \frac{\partial \varphi(s, \tau)}{\partial \tau} d \tau .
\end{aligned}
$$


Proof. The proof of properties (a), (d) and ( $f$ ) is the same as before. To prove the first part of (b), let $\alpha$ be a positive constant and integrate by parts,

$$
\begin{aligned}
\int_{0}^{t+} f d \psi_{t} & =\int_{0}^{t+\alpha} f(\tau) d_{t} \psi(\tau, t) \\
& =f(t+\alpha) \psi(t+\alpha, t)-f(0) \psi(0, t)-\int_{0}^{t+\infty} \psi(\tau, t) d f(\tau) .
\end{aligned}
$$

Since $\psi(\tau, t)=0$ for $\tau>t$ and $f(\tau)=0$ for $\tau<0$, this can be rewritten as

$$
\int_{0}^{t+} f d \psi_{t}=-\int_{0-}^{t} \psi(\tau, t) d f(\tau)=-\int_{0-}^{t} \psi_{t} d f
$$

as asserted. For the second part of (b), integrate by parts and use the fact that $\varphi(s, \tau)=0$ for $\tau<s$.

The proof of $(\mathrm{g})$ is essentially the same as before. To obtain the first and third Riemann integral expansions let $\psi(\tau, t)=\psi(t, t) 1(\tau-t)+\tilde{\psi}(\tau, t)$, where $\tilde{\psi}(, t)$ is continuous on $[0, \infty)$.

To show (e), set the left hand side of the first expansion in (g) equal to zero. The result is a homogeneous linear Volterra integral equation of the second kind. Now apply the discussion following (6.11).

Property (c) can be established by first letting

$$
\theta=\int_{0-}^{t}\left[-\int_{0}^{t+} s \varphi d \psi_{t}\right]_{t} d f=\int_{0-}^{t}\left[-\int_{0}^{t+} \varphi(s, \tau) d_{t} \psi(\tau, t)\right] d f(s)
$$

and then applying the expansions in $(\mathrm{g})$ to give

$$
\begin{aligned}
\theta= & \int_{0-}^{t}\left[\psi(t, t) \varphi(s, t)-\int_{s}^{t} \varphi(s, \tau) \frac{\partial \psi(\tau, t)}{\partial \tau} d \tau\right] d f(s) \\
= & \psi(t, t) \int_{0-}^{t} \varphi(s, t) d f(s)-f(0) \int_{0}^{t} \varphi(0, \tau) \frac{\partial \psi(\tau, t)}{\partial \tau} d \tau \\
& -\int_{0}^{t} \int_{a}^{t} \varphi(a, \tau) \frac{d f(a)}{d a} \frac{\partial \psi(\tau, t)}{\partial \tau} d \tau d a .
\end{aligned}
$$

Interchanging the order of integration and recombining terms in $\theta$ gives

$$
\begin{aligned}
\theta= & \psi(t, t) \int_{0-}^{t} \varphi(s, t) d f(s)-f(0) \int_{0}^{t} \varphi(0, \tau) \frac{\partial \psi(\tau, t)}{\partial \tau} d \tau \\
& -\int_{0}^{t} \int_{0}^{\tau} \varphi(s, \tau) \frac{d f(s)}{d s} \frac{\partial \psi(\tau, t)}{\partial \tau} d s d \tau \\
= & \psi(t, t) \int_{0-}^{t} \varphi(s, t) d f(s)-\int_{0}^{t}\left[\int_{0-}^{t} \varphi(s, \tau) d f(s)\right] \frac{\partial \psi(\tau, t)}{\partial \tau} d \tau \\
= & -\int_{0}^{t+}\left[\int_{0-}^{\tau} \varphi(s, \tau) d f(s)\right] d_{t} \psi(\tau, t) .
\end{aligned}
$$


Equating (6.16) and (6.17) and using property (b) gives the desired result.

The integrals in (6.2 a), (6.3a) reduce to Stieltjes convolutions if $\varphi$ and $\psi$ are independent of $t$. The integrals in (6.14) becomes Stieltjes convolutions if $\psi(\tau, t)$ $=\psi(t-\tau)$ and $\varphi(s, \tau)=\varphi(\tau-s)$. As discussed in Section 5 , there are also the conditions required for the "aging" stress-strain relations (3.12) and (3.17) to reduce to a non-aging one. It is worth noting that if these reductions take place, then the operator algebras defined here reduce to that presented in [1].

\section{References}

[1] Gurtin, M. E., and E. Sternberg: On the Linear Theory of Viscoelasticity. Arch. Rational Mech. Anal. 11, 291 (1962).

[2] MorLand, L. W., and E. H. LeE: Stress Analysis of Linear Viscoelastic Materials with Temperature Variation. Tran. Soe. of Rheol. 4, 233 (1960).

[3] STouffer, D. C., and A. S. Wineman : Linear Viscoelastic Materials with EnvironmentalDependent Properties. Intern. J. Eng. Sci. 9, 193 (1971).

[4] Arutyunyan, N. KH.: Some Problems in the Theory of Creep. (Translated by H. E. Nowottny.) New York: Pergamon Press. 1966.

[5] Sackman, J. L.: Creep of Concrete and Concrete Structures. Proceedings of the Princeton University Conference on Solid Mechanics. Princeton, N.J.: 1963.

[6] Truesdenc, C.: The Elements of Continuum Mechanics. Berlin-Heidelberg-New York: Springer. 1966.

[7] Köntg, H. and J. Metxner: Lineare Systeme und Lineare Transformation. Mathematische Nachrichten 19, 256 (1958).

[8] Rresz, F., and B. Sz-Nagy: Functional Analysis. (Translated by L. Bonon.) New York: Frederick Ungar Pub. 1955.

[9] Sokolnıkoff, J. S.: Mathematical Theory of Elasticity. New York: McGraw-Hill Book Co. 1956.

[10] Rogers, T. G., and A. C. PIPkin: Asymmetric Relaxation and Compliance Matrices in Linear Viscoelasticity. ZAMP 14, 334 (1963).

[11] Apostor, T. M.: Mathematical Analysis. Reading, Mass.: Addison-Wesley. 1964.

[12] Flügae, W.: Viscoelasticity. Waltham, Mass.: Blaisdell. 1967.

[13] SchwarzL, F., and A.J. Stafyerman: Time-Temperature Dependence of Linear Viscoelastic Behavior. J. Appl. Phys. 23, 838 (1952).

[14] Smrthrms, F.: Integral Equations. London: Cambridge University Press. 1958.

\author{
Prof. D. C. Stouffer \\ University of Cincinnati \\ Cincinnati, OH 45221, U.S.A.
}

Prof. A. S. Wineman

University of Michigan Ann Arbor, $M I$ 48104, U.S.A. 